\title{
The Role of NAD+ in Anti-Aging Therapies
}

\author{
Xuqian Liu ${ }^{1}$ and Taosheng Huang ${ }^{1,2 *}$ \\ ${ }^{1}$ Human Aging Research Institute, Nanchang University, China \\ ${ }^{2}$ Division of Human Genetics, Cincinnati Children's Hospital Medical Center, China
}

*Corresponding author: Taosheng Huang, Human Aging Research Institute, Nanchang University, Nanchang 330031 and Division of Human Genetics, Cincinnati Children's Hospital Medical Center, China.

To Cite This Article: Taosheng Huang, The Role of NAD+ in Anti-Aging Therapies. Am J Biomed Sci \& Res. 2019 - 6(5). AJBSR.MS.ID.001080.

DOI: 10.34297/AJBSR.2019.06.001080.

Received: 啙 December 11, 2019 ; Published: 眥 December 20, 2019

\begin{abstract}
The aging process involves accumulation of DNA damage, mitochondrial defects, progressive tissue degeneration and atrophy, and the development of metabolic dysfunction and weakness. Aging is also accompanied by decreased levels of nicotinamide adenine dinucleotide (NAD+), which can result in cell damage and even shorter life spans. $\mathrm{NAD}^{+}$acts as an enzyme cofactor in many essential biological pathways and is a substrate for several regulatory proteins. Many studies have suggested that the upregulation of $\mathrm{NAD}^{+}$precursors can increase levels of $\mathrm{NAD}^{+}$in tissues or cells to delay aging. Clinical trials have been conducted on the safety and efficacy of $\mathrm{NAD}^{+}$. Here we provide a review of $\mathrm{NAD}^{+}$metabolism and its role in aging-related therapy.
\end{abstract}

Keywords: NAD+; Aging; Clinical trials

\section{Introduction}

The aging process is influenced by a variety of factors and considered to be an irreversible process. Aging of an organism is accompanied by metabolic disorders and the impairment of physiological function, as well as the development of age-related diseases [1-4]. There is abundant evidence that $\mathrm{NAD}^{+}$plays an important role in aging, as it is involved in various biological functions and is a key regulator of stress resistance [5,6]. Levels of $\mathrm{NAD}^{+}$steadily decline with age, resulting in altered metabolism and increased disease susceptibility [7-9]. NAD plays a key role in various energy metabolism pathways $[6,10]$. Additionally, $\mathrm{NAD}^{+}$is a cofactor for many enzymes, such as poly (ADP-ribose) polymerases (PARPs), CD38, and sirtuins [11]. Sirtuins are $\mathrm{NAD}^{+}$-dependent histone deacetylase for a wide range of transcriptional regulators $[10,12]$. Overexpression of SIRT1 in the brains of mice has been shown to delay aging [13]. $\mathrm{PARP}$ is a major $\mathrm{NAD}^{+}$-degrading enzyme, which plays diverse roles in many molecular and cellular processes [14]. Inhibition of PARP-1 increases mitochondrial metabolism via modulation of SIRT1 activity [15]. Another $\mathrm{NAD}^{+}$-degrading enzyme, CD38, had been associated with the decline in $\mathrm{NAD}^{+}$levels during aging [16].

Mammalian cells cannot import $\mathrm{NAD}^{+}$in vivo, so they must synthesize it either from tryptophan or the various forms of niacin taken up in the diet including nicotinamide mononucleotide (NMN) and nicotinamide riboside (NR) [17-20]. Recently, it was found in mice that supplementing with $\mathrm{NAD}^{+}$precursors (including $\mathrm{NMN}$, $\mathrm{NR}$, and nicotinamide) or inhibiting the activity of $\mathrm{NAD}^{+}$-consuming enzymes can increase the level of $\mathrm{NAD}^{+}$in tissues and improve energy metabolism, thereby delaying aging and extending healthy life [15,21-24].

Currently, the anti-aging activity of $\mathrm{NAD}^{+}$precursors is primarily evaluated through measurement of aging markers in mouse behavior, accumulation of DNA damage, and mitochondrial activity. RNA sequencing has also been used to identify genes and pathways involved in the anti-aging mechanisms of $\operatorname{NAD}^{+}[20,22,25,29]$. Furthermore, recent research has shown that biological age can be measured by analyzing the 353 DNA methylation sites of the Horvath clock [30,31].

\section{NAD ${ }^{+}$Biosynthesis-Salvage Pathway}

In vivo, $\mathrm{NAD}^{+}$is an essential cofactor of dehydrogenase [32,33]. Nicotinamide coenzyme is an electron carrier which plays an important role in various oxidation-reduction reactions. Therefore $\mathrm{NAD}^{+}$is a cofactor of many key enzymes in glycolysis, the tricarboxylic acid cycle, and oxidative phosphorylation [34]. Age-associated 
decline in $\mathrm{NAD}^{+}$availability has an important effect on the aging process of many species $[8,35,36]$. There are three pathways for the synthesis of $\mathrm{NAD}^{+}$in cells, involving many different precursors [3739]. Here we focus on the salvage pathway, which is important from a translational research perspective because it is the main source of $\mathrm{NAD}^{+}[40,41]$.

There are three pathways for the synthesis of $\mathrm{NAD}^{+}$in cells:

a. de novo from tryptophan;

b. from nicotinic acid via the Preiss-Handler pathway; and

c. from nicotinamide (NAM) via the salvage pathway [37].

NAM itself is a by-product of $\mathrm{NAD}^{+}$-degrading enzymes such as sirtuins and PARP. As shown in Figure 1, the first step of the sal- vage pathway is catalyzed by nicotinamide phosphoribosyl-transferase (NAMPT), which converts nicotinamide and 5-phosphoribosyl-1-pyrophosphate into NMN [42]. Subsequently, nicotinamide mononucleotide adenylyltransferase (NMNAT) produces $\mathrm{NAD}^{+}$ from NMN and ATP $[43,44]$. NR can be converted by nicotinamideriboside kinase (NRK) into NMN which participating in the Salvage pathway [45]. NAMPT is the rate-limiting enzyme of the salvage pathway [42]. It has been hypothesized that reduced NAD ${ }^{+}$synthesis is one of the causes of lower $\mathrm{NAD}^{+}$levels with aging, and this may be due to decreased activity of NAMPT $[42,46]$. Indeed, NAMPT levels are known to decline with age in many types of tissues [4749], whereas exercise increases skeletal muscle NAMPT expression [50].

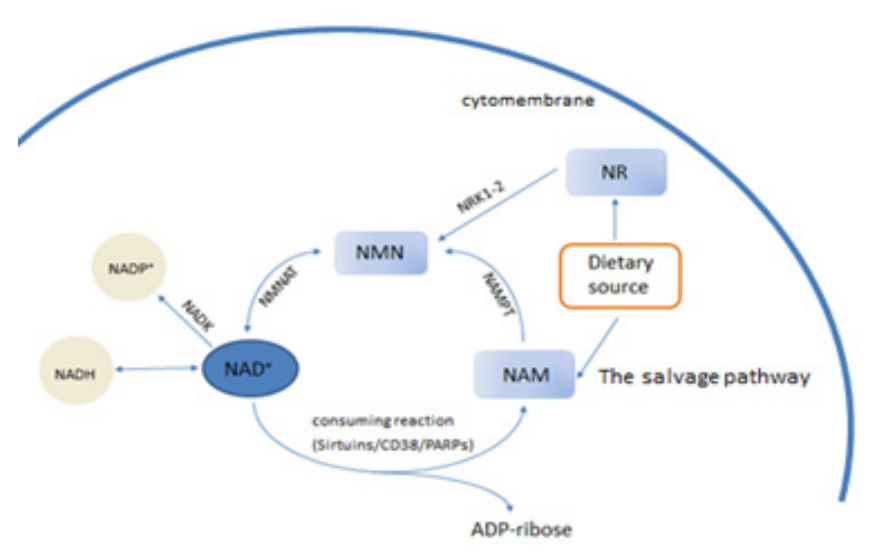

Figure 1: The Salvage pathway NAM and NR are the main precursor for the salvage pathway.

In mammals, NAMPT has two different forms: intra- and extracellular [51]. The intracellular form is the one that participates in the salvage pathway of $\mathrm{NAD}^{+}$synthesis [42], while the extracellular form likely functions as a circulating cytokine [52]. Studies have shown that secretion of NAMPT is regulated by SIRT1 in vivo $[53,54]$, and SIRT1 activity in turn depends on NAMPT which regulates level of NAD+ [55]. Increasing level of NAMPT may delay aging of individuals via SIRT1-dependent pathways [56]. NAMPT has been shown to regulate osteoblast differentiation in primary culture of mouse bone marrow-derived mesenchymal stem cells via NAD+-SIRT1 pathway. NAMPT deficiency may increase the risk of bone aging or fractures [48,57-59].

Levels of NAMPT significantly decrease with age in mice and humans $[60,61]$. Adipose tissue-specific overexpression of NAMPT in aged mice resulted in increased levels of circulating eNAMPT, increased levels of $\mathrm{NAD}^{+}$in multiple tissues, and extended lifespan [60].

In mammals NMNAT is the central enzyme of the NAD biosynthetic pathway $[43,62]$. There are three isoforms, NMNAT1, NMNAT2, and NMNAT3, encoded by different genes and localized to nucleus, Golgi apparatus, and mitochondria, respectively [43]. NMNAT1 directly control SIRT1 deacetylase activity at a set of target gene promoters [63]. Homozygous knockout of Nmnat1 in mice results in embryonic death [64]. Low levels of NMNAT2, highly expressed in the brain and nervous system, could impair axon regeneration as well as axon survival in aging and disease $[65,66]$. NMNAT3 has been identified as the rate-limiting enzyme for mitochondrial $\mathrm{NAD}^{+}$biosynthesis [67]. In addition, down-regulation of NMNAT3 gene expression in cells significantly impairs the capacity for mitochondrial respiration, suggesting that NMNAT3 plays a key role in mitochondrial $\mathrm{NAD}^{+}$homeostasis [68].

\section{Anti-aging effects of NAD ${ }^{+}$}

Through its role as a substrate for sirtuins, CD38, and PARP, $\mathrm{NAD}^{+}$regulates a variety of cellular process including energy metabolism, DNA damage repair, gene expression, and oxidative stress response $[11,34,69,70]$.

\section{a. The Sirtuins Pathway}

Sirtuins are evolutionarily conserved $\mathrm{NAD}^{+}$-dependent deacetylases. Increasing sirtuin expression has been shown to affect lifes- 
pan across various species [13,36,71,72]. Sirtuins have received significant attention since the discovery that the increased sirtuin silent information regulator 2 (Sir2) can extend yeast lifespan [72]. The closest mammalian homologue of this regulator is SIRT1[69], mainly localized in the nucleus but also present in cytosol [73]. Its nuclear export signal allows shuttling to the cytosol under specific circumstance [12]. It has been shown in vivo that NAD+-SIRT1 signaling promotes mitochondrial activity [25]. Previous research has suggested that increasing SIRT1 in the brain, especially in the dorsomedial and lateral hypothalamic nuclei, can delay aging and extend lifespan in mice [13]. More recently, heart-specific overexpression of Sirt1 was found to delay aging and protect against oxidative stress in the heart [74]. NR-SIRT1 signaling can inhibit cardiac stem cell senescence by improving mitochondrial function and muscle stem cell function, thereby enhancing life span in mice [25]. More research is needed to determine whether increased level of $\mathrm{NAD}^{+}$in vivo can improve SIRT1 activity, thereby delaying aging [75].

\section{b. The PARPs Pathway}

PARPs are expressed by most eukaryotic cells and are involved in DNA damage detection and repair, cell death pathways and so on [14]. Aging is associated with an accumulation of DNA damage [76]. Depletion of $\mathrm{NAD}^{+}$is involved in cell death through PARP-1 [70]. Although this enzyme plays an important role in cells, over-activation of PARP-1 can lead to depletion of $\mathrm{NAD}^{+}$, reduction of ATP, reducing the activity of SIRT1, loss of mitochondrial function, and even cell death $[70,77,78]$. Increased level of $\mathrm{NAD}^{+}$, when SIRT1 is intact, can reduce the cell death caused by activation of PARP-1 in cardiac myocyte [79].

\section{c. CD38 and NAD ${ }^{+}$}

CD38 is a multi-functional protein. Studies have shown that CD38 is the NADase in mammalian tissues [80,81]. It is thought to contribute to the age-related decline in NAD levels $[23,80,82]$. CD38 also acts as an antigen for B-lymphocyte activation and as an ecto-enzyme in endothelial and inflammatory cells [82,83]. Senescent cells are known to express small molecules including secreted cytokines, growth factors, and extracellular matrix modifiers to promote chronic sterile inflammation and fibrosis. This reaction process, known as the senescence-associated secretory phenotype [84-86], involves secretion of factors by senescent cells which induce the expression of CD38 in non-senescent cells [82,87]. This increased CD38 activity can disrupt the fine balance between $\mathrm{NAD}^{+}$ and its reduced form, NADH, within a cell [82]. Recently, the small molecule CD38 inhibitor 78c was shown to reverse the age-related loss of $\mathrm{NAD}^{+}[28,83]$. By increasing tissue levels of $\mathrm{NAD}^{+}, 78 \mathrm{c}$ may be able to ameliorate metabolic disorder and other disruptions involved in the aging process. In addition, animals treated with 78c show activation of longevity genes, which inhibit DNA damage [28].

$\mathrm{NAD}^{+}$and $\mathrm{NADH}$ are in dynamic equilibrium within the cell [75]. Intracellular $\mathrm{NAD}^{+}$can be increased in vivo through oral ad- ministration of $\mathrm{NAD}^{+}$precursor or by inhibiting the degradation of $\mathrm{NAD}^{+}[15,23,88]$. Regulation of the $\mathrm{NAD}^{+} / \mathrm{NADH}$ ratio in this way can improve mitochondrial function and has been shown to treat senile deafness in elderly mice [89].

\section{$\mathrm{NAD}^{+}$Repletion and Aging}

One of the major causes of aging is progressive tissue degeneration and atrophy due to reduced somatic or stem cell function $[22,90,91]$. Adult stem cells are not only essential in continuously proliferating tissues (such as hematopoietic, intestinal, and skin systems) but also in normally quiescent tissues (such as skeletal muscle and the brain) that require regeneration after damage or exposure to disease [92]. NR supplementation improved metabolic function in muscle and neural stem cells, in both young and old mice, thereby increasing lifespan [25]. NR treatment has also been shown to rejuvenate stem cells from aged mice and restore the impaired ability to repair gut damage [22]. Previous studies have shown that DNA damage of nerve cells, nerve stem cells, and muscle stem cells in mice can be reduced by NR supplementation $[25,93]$. NR has also been shown to ameliorate mitochondrial dysfunction and enhance oxidative metabolism in obese mice [94,95] and prolong the lifespan of mice through neuronal DNA repair and mitochondrial quality improvement $[96,97]$.

Supplementation with NMN can restore age-related capillary rarefaction and increase blood flow in elderly mice, and it maybe a novel therapy to restore SIRT1 activity and reverse age-related arterial dysfunction by reducing oxidative stress $[98,99]$. Mitochondrial disorders due to impaired oxidative phosphorylation (OXPHOS) are a cause of aging [100]. Long-term treatment with NMN in elderly C57BL/6J mice can improve metabolic dysfunction and ameliorate age-associated physiological decline [20]. NMN can also restore mitochondrial function, prevent neural death, and delay cognitive decline in a mouse model of Alzheimer's disease [101,102]. Supplementation with NAM was shown to improve blood sugar levels and metabolic capacity in HFD-fed mice. However, it had no effect on lifespan [103].

There are data showing that supplementation with $\mathrm{NAD}^{+}$precursors enhanced the mitochondrial function of cells or stem cells in a SIRT1-dependent manner [25,94]. Furthermore, supplementing $\mathrm{NAD}^{+}$precursors in elderly mice improved mitochondrial function in hematopoietic stem cells and muscle stem cells, as well as extended lifespan $[25,104]$. NMN specifically was found to enhance the biological activity of mesenchymal stromal cells through the upregulation of SIRT1, thereby stimulating osteogenesis of the cells and protecting bone from aging to delay the aging of mice [105]. In elderly mice, NMN treatment improved capillary density through the $\mathrm{NAD}^{+}-\mathrm{H}_{2} \mathrm{~S}$ signaling network to increase blood flow, endurance, and physiological status $[27,29,106]$.

Inhibition of some $\mathrm{NAD}^{+}$-degrading enzymes could also lead to increased levels of $\mathrm{NAD}^{+}[15,23]$. CD38, PARPs, and SARM1 all 
degrade NAD ${ }^{+}$inside the cell $[23,77,80,107]$. The activity of CD38 increases with aging, contributing to the age-related decline in $\mathrm{NAD}^{+}$[16]. Several small-molecule inhibitors of CD38 have been described [83,108-110]. Thiazoloquin(az)olin(on)e is one such inhibitor which could potentially be used as a therapeutic agent to increase intracellular $\mathrm{NAD}^{+}$level [28]. Inhibition of PARP1 has recently been reported to correct mitochondrial impairment [111,112] and has strong metabolic implications through its modulation of SIRT1 activity [15].

\section{Measure Biological Age}

Thus far, the anti-aging activity of $\mathrm{NAD}^{+}$is mainly examined using RNA sequencing and gene set enrichment analysis to identify pathways of candidate biomarkers [20,22,25]. However, there is not yet a gold standard for aging biomarkers. The DNA clock may offer a better objective biomarker for the study of aging [30,31]. DNA methylation plays a critical role in the regulation of gene transcription [113-118]. Senescence can be predicted and evaluated by detecting cytosine-5 methylation within $\mathrm{CpG}$ dinucleotides [30,31]. These age-related $\mathrm{CpG}$ characteristics are independent of gender or tissue type. Recent research has shown that biological age can be approximated by measuring levels of DNA methylation, a process known as the Horvath (or DNA) clock [30,31,119,120]. Age-related DNA methylation was first described for humans after cross comparison of thousands of $\mathrm{CpG}$ sites in Illumina Bead Chip microarray data $[121,122]$. Many of these age-associated CpG sites were then used as epigenetic age-predictors [30,31,120,123]. Three hundred and fifty-three unique CpG sites were found to be predictive of biological age, independent of chromatin status or tissue source $[30,31]$.

Petkovich et al. developed a robust predictor of mouse biological age based on $90 \mathrm{CpG}$ sites derived from partial blood DNA methylation profiles [124]. Stubbs et al. further developed a multi-tissue predictor to estimate age based on DNA methylation at 329 unique $\mathrm{CpG}$ sites from various different mouse tissues [125]. One group claims to have found three methylation sites, Prima1, Hsf4, and Kcns1, which are enough to predict biological age in mice [126]. However, this study has yet to be replicated. The most accurate clock results from applying elastic net regression to all CpGs for multi-tissue in mice [127].

Together these studies suggest that the DNA clock provides an objective biomarker for the study of aging [30,31]. Recently, metformin has shown that reversed subject's biological age, based on assessment of Horvath clock [128,129]. Its use will allow us to examine the anti-aging effectiveness of $\mathrm{NAD}^{+}$and its precursors more objectively and accurately.

\section{Clinical Research}

$\mathrm{NAD}^{+}$precursors can be delivered orally to humans or animals to alter the dynamic balance of $\mathrm{NAD}^{+} / \mathrm{NADH}$ in vivo $[24,130]$. Preliminary clinical studies in humans showed that NR supple- mentation could improve muscle $\mathrm{NAD}^{+}$metabolism in the elderly $[131,132]$. Healthy volunteers, who underwent an 8-day course of NR, with doses increasing from $250 \mathrm{mg}$ to $1000 \mathrm{mg}$, showed increased levels of circulating $\mathrm{NAD}^{+}$and experienced no adverse side effects [133]. Similarly, NR supplementation increased NADH and NADPH levels and improved exercise performance in elderly subjects [134]. Therefore, NMN is considered safe in clinical trials [135]. However, high dose supplementation with $\mathrm{NAD}^{+}$precursors may increase rates of glycolysis and mitochondrial respiratory metabolism, thereby promoting the secretion of proinflammatory cytokines in cells [136]. Thus, use of supplements should be carefully observed to ensure that they strike a proper balance between anti-aging effects and potential detrimental effects.

\section{Conclusions}

$\mathrm{NAD}^{+}$is a cofactor for many important enzymes. Reduced levels of $\mathrm{NAD}^{+}$have been associated with aging. Evidence suggests that supplementation with $\mathrm{NAD}^{+}$precursors, or inhibition of $\mathrm{NAD}^{+}$degradation, could improve metabolic function. While supplementation with $\mathrm{NAD}^{+}$precursors has been found to delay aging in mice, anti-aging effects of $\mathrm{NAD}^{+}$have yet to be demonstrated in human subjects. Use of a more accurate biomarker for aging, such as the DNA methylation clock, will significantly advance the field. Recently, several human clinical trials have been initiated.

\section{References}

1. Fraga MF, Agrelo R, Esteller M (2007) Crosstalk between aging and cancer: the epigenetic language. Ann N Y Acad Sci 1100: 60-74.

2. Fraga MF, Esteller M (2007) Epigenetics and aging: the targets and the marks. Trends Genet 23(8): 413-418.

3. Braidy N, Grant R, Sachdev PS (2018) Nicotinamide adenine dinucleotide and its related precursors for the treatment of Alzheimer's disease. Curr Opin Psychiatry 31(2): 160-166.

4. Fuellen G, Jansen L, Cohen AA, Walter Luyten, Manfred Gogol, et al. (2019) Health and Aging: Unifying Concepts, Scores, Biomarkers and Pathways. Aging Dis 10(4): 883-900.

5. Hosseini L, Vafaee MS, Mahmoudi J, Badalzadeh R (2019) Nicotinamide adenine dinucleotide emerges as a therapeutic target in aging and ischemic conditions. Biogerontology 20(4): 381-395.

6. Ying $\mathrm{W}$ (2008) NAD+/NADH and NADP+/NADPH in cellular functions and cell death: regulation and biological consequences. Antioxid Redox Signal 10(2): 179-206.

7. Zhang M, Ying W (2019) NAD (+) Deficiency Is a Common Central Pathological Factor of a Number of Diseases and Aging: Mechanisms and Therapeutic Implications. Antioxid Redox Signal 30(6): 890-905.

8. Mouchiroud L, Houtkooper RH, Moullan N, Katsyuba E, Ryu D, et al. (2013) The NAD (+)/Sirtuin Pathway Modulates Longevity through Activation of Mitochondrial UPR and FOXO Signaling. Cell 154(2): 430441.

9. Stein LR, Imai S (2014) Specific ablation of Nampt in adult neural stem cells recapitulates their functional defects during aging. EMBO J 33(12): 1321-1340.

10. Houtkooper RH, Pirinen E, Auwerx J (2012) Sirtuins as regulators of metabolism and healthspan. Nat Rev Mol Cell Biol 3(4): 225-238.

11. Katsyuba E, Auwerx J (2017) Modulating NAD (+) metabolism, from bench to bedside. EMBO J 36(18): 2670-2683. 
12. Tanno M, Sakamoto J, Miura T, Shimamoto K, Horio Y (2007) Nucleocytoplasmic shuttling of the NAD+-dependent histone deacetylase SIRT1. J Biol Chem 282(9): 6823-6832.

13. Satoh A, Brace CS, Rensing N, Cliften P, Wozniak DF, et al. (2013) Sirt1 extends life span and delays aging in mice through the regulation of Nk2 homeobox 1 in the DMH and LH. Cell Metab 18(3): 416-430.

14. Kim MY, Zhang T, Kraus WL (2005) Poly (ADP-ribosyl)ation by PARP1: 'PAR-laying' NAD+ into a nuclear signal. Genes Dev 2005; 19(17): 1951-1967.

15. Bai P, Canto C, Oudart H, Attila Brunyánszki, Yana Cen, et al. (2011) PARP-1 inhibition increases mitochondrial metabolism through SIRT1 activation. Cell Metab 13(4): 461-468.

16. Camacho Pereira J, Tarrago MG, Chini CCS, Nin V, Escande C, et al (2016) CD38 Dictates Age-Related NAD Decline and Mitochondrial Dysfunction through an SIRT3-Dependent Mechanism. Cell Metab 23(6): 1127-1139.

17. Trammell SA, Yu L, Redpath P, Migaud ME, Brenner C (2016) Nicotinamide Riboside Is a Major NAD+ Precursor Vitamin in Cow Milk. J Nutr 146(5): 957-963.

18. Bogan KL, Brenner C (2008) Nicotinic acid, nicotinamide, and nicotinamide riboside: a molecular evaluation of NAD+ precursor vitamins in human nutrition. Annu Rev Nutr 28: 115-130.

19. Ummarino S, Mozzon M, Zamporlini F, Amici A, Mazzola F, et al. (2017) Simultaneous quantitation of nicotinamide riboside, nicotinamide mononucleotide and nicotinamide adenine dinucleotide in milk by a novel enzyme-coupled assay. Food Chem 221: 161-168.

20. Mills KF, Yoshida S, Stein LR, Grozio A, Kubota S, et al. (2016) LongTerm Administration of Nicotinamide Mononucleotide Mitigates AgeAssociated Physiological Decline in Mice. Cell Metab 24(6): 795-806.

21. Chaturvedi P, Tyagi SC (2018) NAD (+): A big player in cardiac and skeletal muscle remodeling and aging. J Cell Physiol 233(3): 18951896

22. Igarashi M, Miura M, Williams E, Jaksch F, Kadowaki T, et al. (2019) NAD (+) supplementation rejuvenates aged gut adult stem cells. Aging Cell 18(3): e12935.

23. Chini EN, Chini CCS, Espindola Netto JM, de Oliveira GC, van Schooten W (2018) The Pharmacology of CD38/NADase: An Emerging Target in Cancer and Diseases of Aging. Trends Pharmacol Sci 39(4): 424-436.

24. Yoshino J, Baur JA, Imai SI (2018) NAD (+) Intermediates: The Biology and Therapeutic Potential of NMN and NR. Cell Metab 2018; 27(3): 513-528.

25. Zhang H, Ryu D, Wu Y, Gariani K, Wang X, et al. (2016) NAD+ repletion improves mitochondrial and stem cell function and enhances life span in mice. Science 352(6292): 1463-1443.

26. Tarantini S, Valcarcel-Ares MN, Toth P, Yabluchanskiy A, Tucsek Z, et al. (2019) Nicotinamide mononucleotide (NMN) supplementation rescues cerebromicrovascular endothelial function and neurovascular coupling responses and improves cognitive function in aged mice. Redox Biol 24: 101192

27. Das A, Huang GX, Bonkowski MS, Longchamp A, Li C, et al. (2018) Impairment of an Endothelial NAD (+)-H2S Signaling Network Is a Reversible Cause of Vascular Aging. Cell 173(1): 74-89.

28. Tarrago MG, Chini CCS, Kanamori KS, Warner GM, Caride A, et al. (2018) A Potent and Specific CD38 Inhibitor Ameliorates Age-Related Metabolic Dysfunction by Reversing Tissue NAD (+) Decline. Cell Metab 27(5): 1081-1095

29. Das A, Huang GX, Bonkowski MS et al. (2019) Impairment of an Endothelial NAD (+)-H2S Signaling Network Is a Reversible Cause of Vascular Aging. Cell 176: 944-945.

30. Horvath S (2013) DNA methylation age of human tissues and cell types. Genome Biol 14(10): R115.
31. Horvath S (2015) Erratum to: DNA methylation age of human tissues and cell types. Genome Biol 16: 96.

32. Xiao W, Wang RS, Handy DE, Loscalzo J (2018) NAD(H) and NADP(H) Redox Couples and Cellular Energy Metabolism. Antioxid Redox Signal 28(3): 251-272.

33. Yaku K, Okabe K, Nakagawa T (2018) NAD metabolism: Implications in aging and longevity. Ageing Res Rev 47: 1-17.

34. Berger F, Ramirez-Hernandez $M H$, Ziegler $M$. The new life of a centenarian: signalling functions of NAD(P). Trends Biochem Sci 29(3): 111-118.

35. Imai SI (2016) The NAD World 2.0: the importance of the inter-tissue communication mediated by NAMPT/NAD $(+) /$ SIRT1 in mammalian aging and longevity control. NPJ Syst Biol Appl 2: 16018.

36. Imai S, Guarente L (2014) NAD+ and sirtuins in aging and disease. Trends Cell Biol 24(8): 464-471.

37. Verdin E (2015) NAD (+) in aging, metabolism, and neurodegeneration. Science 350(6265): 1208-1213.

38. Chi Y, Sauve AA (2013) Nicotinamide riboside, a trace nutrient in foods, is a vitamin B3 with effects on energy metabolism and neuroprotection. Curr Opin Clin Nutr Metab Care 16(6): 657-661.

39. Bieganowski P, Brenner C (2004) Discoveries of nicotinamide riboside as a nutrient and conserved NRK genes establish a Preiss-Handler independent route to NAD+ in fungi and humans. Cell 117(4): 495502 .

40. Kennedy BE, Sharif T, Martell E, Dai C, Kim Y, et al. (2016) NAD (+) salvage pathway in cancer metabolism and therapy. Pharmacol Res 114: 274-283.

41. Houtkooper RH, Canto C, Wanders RJ, Auwerx J (2010) The secret life of NAD+: an old metabolite controlling new metabolic signaling pathways. Endocr Rev 31(2): 194-223.

42. Revollo JR, Grimm AA, Imai S (2004) The NAD biosynthesis pathway mediated by nicotinamide phosphoribosyltransferase regulates Sir2 activity in mammalian cells. J Biol Chem 279(49): 50754-50763.

43. Berger F, Lau C, Dahlmann M, Ziegler M (2005) Subcellular compartmentation and differential catalytic properties of the three human nicotinamide mononucleotide adenylyltransferase isoforms. J Biol Chem 280(43): 36334-36341.

44. Schweiger M, Hennig K, Lerner F, Niere M, Hirsch-Kauffmann M, et al. (2001) Characterization of recombinant human nicotinamide mononucleotide adenylyl transferase (NMNAT), a nuclear enzyme essential for NAD synthesis. FEBS Lett 492(1-2): 95-100.

45. Canto C, Menzies KJ, Auwerx J (2015) NAD (+) Metabolism and the Control of Energy Homeostasis: A Balancing Act between Mitochondria and the Nucleus. Cell Metab 22(1): 31-53.

46. van der Veer E, Ho C, O’Neil C, Barbosa N, Scott R, et al. (2007) Extension of human cell lifespan by nicotinamide phosphoribosyltransferase. J Biol Chem 282(15): 10841-10845.

47. Liu LY, Wang F, Zhang XY, Huang P, Lu YB, et al. (2012) Nicotinamide phosphoribosyltransferase may be involved in age-related brain diseases. PLoS One 7(10): e44933.

48. Frederick DW, Loro E, Liu L, Davila A Jr, Chellappa K, et al. (2016) Loss of NAD Homeostasis Leads to Progressive and Reversible Degeneration of Skeletal Muscle. Cell Metab 24(2): 269-282.

49. Mukherjee S, Chellappa K, Moffitt A, Ndungu J, Dellinger RW, et al (2017) Nicotinamide adenine dinucleotide biosynthesis promotes liver regeneration. Hepatology 65(2): 616-630.

50. Costford SR, Bajpeyi S, Pasarica M, Albarado DC, Thomas SC, et al (2010) Skeletal muscle NAMPT is induced by exercise in humans. Am J Physiol Endocrinol Metab 298(1): E117-E126. 
51. Revollo JR, Korner A, Mills KF, Satoh A, Wang T, et al. (2007) Nampt/ $\mathrm{PBEF} /$ Visfatin regulates insulin secretion in beta cells as a systemic NAD biosynthetic enzyme. Cell Metab 6(5): 363-375.

52. Imai S (2009) Nicotinamide phosphoribosyltransferase (Nampt): a link between NAD biology, metabolism, and diseases. Curr Pharm Des 15(1): 20-28.

53. Yoon MJ, Yoshida M, Johnson S, Takikawa A, Usui I, et al. (2015) SIRT1-Mediated eNAMPT Secretion from Adipose Tissue Regulates Hypothalamic NAD+ and Function in Mice. Cell Metab 21(5): 706-717.

54. Nakahata Y, Sahar S, Astarita G, Kaluzova M, Sassone-Corsi P (2009) Circadian control of the NAD+ salvage pathway by CLOCK-SIRT1. Science 324(5927): 654-657

55. Wang LF, Wang XN, Huang CC, Hu L, Xiao YF, et al. (2017) Inhibition of NAMPT aggravates high fat diet-induced hepatic steatosis in mice through regulating Sirt1/AMPKalpha/SREBP1 signaling pathway. Lipids Health Dis 16(1): 82.

56. Koltai E, Szabo Z, Atalay M, Boldogh I, Naito H, et al. (2010) Exercise alters SIRT1, SIRT6, NAD and NAMPT levels in skeletal muscle of aged rats. Mech Ageing Dev 131(1): 21-28.

57. He X, He J, Shi Y, Pi C, Yang Y, et al. (2017) Nicotinamide phosphoribosyltransferase (Nampt) may serve as the marker for osteoblast differentiation of bone marrow-derived mesenchymal stem cells. Exp Cell Res 352(1): 45-52.

58. Ma C, Pi C, Yang Y, Lin L1, Shi Y, et al. (2017) Nampt Expression Decreases Age-Related Senescence in Rat Bone Marrow Mesenchymal Stem Cells by Targeting Sirt1. PLoS One 12(1): e0170930.

59. Li Y, He X, Li Y, He J, Anderstam B, et al. (2011) Nicotinamide phosphoribosyltransferase (Nampt) affects the lineage fate determination of mesenchymal stem cells: a possible cause for reduced osteogenesis and increased adipogenesis in older individuals. J Bone Miner Res 26(11): 2656-2664.

60. Yoshida M, Satoh A, Lin JB, Mills KF, Sasaki Y, et al. (2019) Extracellular Vesicle-Contained eNAMPT Delays Aging and Extends Lifespan in Mice. Cell Metab 30(2): 329-342.

61. Yoshino J, Mills KF, Yoon MJ, ImaiS (2011) Nicotinamidemononucleotide, a key $\mathrm{NAD}(+)$ intermediate, treats the pathophysiology of diet- and age-induced diabetes in mice. Cell Metab 14(4): 528-536.

62. Magni G, Amici A, Emanuelli M, Orsomando G, Raffaelli N, et al. (2004) Enzymology of NAD+ homeostasis in man. Cell Mol Life Sci 61(1): 1934.

63. Zhang T, Berrocal JG, Frizzell KM, Gamble MJ, DuMond ME, et al. (2009) Enzymes in the NAD+ salvage pathway regulate SIRT1 activity at target gene promoters. J Biol Chem 284(30): 20408-20417.

64. Conforti L, Janeckova L, Wagner D, Mazzola F, Cialabrini, et al. (2011) Reducing expression of NAD+ synthesizing enzyme NMNAT1 does not affect the rate of Wallerian degeneration. FEBS J 278(15): 2666-2679.

65. Gilley J, Adalbert R, Yu G, Coleman MP (2013) Rescue of peripheral and CNS axon defects in mice lacking NMNAT2. J Neurosci 33(33): 1341013424.

66. Hicks AN, Lorenzetti D, Gilley J, Lu B, Andersson KE, et al. (2012) Nicotinamide mononucleotide adenylyltransferase 2 (Nmnat2) regulates axon integrity in the mouse embryo. PLoS One 7(10): e47869.

67. Yue Z, Ma Y, You J, Li Z, Ding Y, et al. (2016) NMNAT3 is involved in the protective effect of SIRT3 in Ang II-induced cardiac hypertrophy. Exp Cell Res 347(2): 261-273.

68. VanLinden MR, Dolle C, Pettersen IK, Kulikova VA, Niere M, et al. (2015) Subcellular Distribution of NAD+ between Cytosol and Mitochondria Determines the Metabolic Profile of Human Cells. J Biol Chem 290(46): 27644-27659.
69. Herranz D, Munoz-Martin M, Canamero M, Francisca Mulero, Barbara Martinez Pastor, et al. (2010) Sirt1 improves healthy ageing and protects from metabolic syndrome-associated cancer. Nat Commun 1: 3.

70. Julio C Morales, Li L, Farjana J Fattah, Ying Dong, Erik A Bey, et al. (2014) Review of Poly (ADP-ribose) Polymerase (PARP) Mechanisms of Action and Rationale for Targeting in Cancer and Other Diseases. Crit Rev Eukaryot Gene Expr 24(1) :15-28.

71. Rogina B, Helfand SL (2004) Sir2 mediates longevity in the fly through a pathway related to calorie restriction. Proc Natl Acad Sci U S A 101(45): 15998-16003.

72. Matt Kaeberlein MM, Leonard Guarente (1999) The SIR2/3/4 complex and SIR2 alone promote longevity in Saccharomyces cerevisiae by two different mechanisms. Genes Dev 13(19): 2570-2580.

73. Tang BL (2016) Sirt1 and the Mitochondria. Mol Cells 39(2): 87-95.

74. Alcendor RR, Gao S, Zhai P, Zablocki D, Holle E, et al. (2007) Sirt1 regulates aging and resistance to oxidative stress in the heart. Circ Res 100(10): 1512-1521.

75. Sultani G, Samsudeen AF, Osborne B, Turner N (2017) NAD (+): A key metabolic regulator with great therapeutic potential. J Neuroendocrinol 29(10).

76. Nakamura AJ, Chiang YJ, Hathcock KS, Horikawa I, Sedelnikova OA, et al. (2008) Both telomeric and non-telomeric DNA damage are determinants of mammalian cellular senescence. Epigenetics Chromatin 1(1): 6

77. Hou WH, Chen SH, Yu X (2019) Poly-ADP ribosylation in DNA damage response and cancer therapy. Mutat Res 780: 82-91.

78. Sas K, Szabo E, Vecsei L (2018) Mitochondria, Oxidative Stress and the Kynurenine System, with a Focus on Ageing and Neuroprotection. Molecules 23(1).

79. Pillai JB, Isbatan A, Imai S, Gupta MP (2005) Poly (ADP-ribose) polymerase-1-dependent cardiac myocyte cell death during heart failure is mediated by NAD+ depletion and reduced Sir2alpha deacetylase activity. J Biol Chem 280(52): 43121-43130.

80. Aksoy P, Escande C, White TA, Thompson M, Soares S, et al. (2006) Regulation of SIRT 1 mediated NAD dependent deacetylation: a novel role for the multifunctional enzyme CD38. Biochem Biophys Res Commun 349(1): 353-359.

81. Aksoy P, White TA, Thompson M, Chini EN, Benech JC, et al. (2006) Regulation of intracellular levels of NAD: a novel role for CD38. Biochem Biophys Res Commun 345(1): 1386-1392.

82. Chini C, Hogan KA, Warner GM, Tarragó MG, Peclat TR, et al. (2019) The NADase CD38 is induced by factors secreted from senescent cells providing a potential link between senescence and age-related cellular NAD(+) decline. Biochem Biophys Res Commun 513(2): 486-493.

83. Dong M, Si YQ, Sun SY, Pu XP, Yang ZJ, et al. (2011) Design, synthesis and biological characterization of novel inhibitors of CD38. Org Biomol Chem 9(9): 3246-3257.

84. Biran A, Zada L, Abou Karam P, Vadai E, Roitman L, et al. (2017) Quantitative identification of senescent cells in aging and disease. Aging Cell 16(4): 661-671.

85. Wiley CD, Velarde MC, Lecot P, Liu S, Sarnoski EA, et al. (2016) Mitochondrial Dysfunction Induces Senescence with a Distinct Secretory Phenotype. Cell Metab 23(2): 303-314.

86. Rodier F, Coppé JP, Patil CK, Hoeijmakers WA, Muñoz DP, et al. (2009) Persistent DNA damage signalling triggers senescence-associated inflammatory cytokine secretion. Nat Cell Biol 11: 973-979.

87. Xu M, Pirtskhalava T, Farr JN, Weigand BM, Palmer AK, et al. (2018) Senolytics improve physical function and increase lifespan in old age. Nat Med 24: 1246-1256. 
88. Belenky P, Bogan KL, Brenner C (2007) NAD+ metabolism in health and disease. Trends Biochem Sci 32: 12-19.

89. Hyung-Jin Kim, Wa Cao, Gi-Su Oh, Seung Hoon Lee, AiHua Shen, et al. (2018) Augmentation of cellular NAD(+) by NQ01 enzymatic action improves age-related hearing impairment. Aging Cell 18(5): e13016.

90. Sharpless NE, DePinho RA (2007) How stem cells age and why this makes us grow old. Nat Rev Mol Cell Biol 8: 703-713.

91. Lopez-Otin C, Blasco MA, Partridge L, Manuel Serrano, Guido Kroemer, et al. (2013) The hallmarks of aging. Cell 153: 1194-1217.

92. Wagers AJ, Weissman IL (2004) Plasticity of adult stem cells. Cell 116: 639-648.

93. Hou Y, Lautrup S, Cordonnier S, Wang Y, Croteau DL, et al. (2018) NAD supplementation normalizes key Alzheimer's features and DNA damage responses in a new AD mouse model with introduced DNA repair deficiency. 115: E1876-E1885.

94. Cantó C, Houtkooper RH, Pirinen E, Youn DY, Oosterveer MH, et al. (2012) The $\mathrm{NAD}(+)$ precursor nicotinamide riboside enhances oxidative metabolism and protects against high-fat diet-induced obesity. Cell Metab 15: 838-847.

95. Gariani K, Menzies KJ, Ryu D, Wegner CJ, Wang X, et al. (2016) Eliciting the mitochondrial unfolded protein response by nicotinamide adenine dinucleotide repletion reverses fatty liver disease in mice. Hepatology 63: 1190-1204.

96. Fang EF, Kassahun H, Croteau DL, Scheibye-Knudsen M, Marosi K, et al. (2016) NAD(+) Replenishment Improves Lifespan and Healthspan in Ataxia Telangiectasia Models via Mitophagy and DNA Repair. Cell Metab 24: 566-581.

97. Guarente L (2016) Overcoming ATM Deficiency by Activating the $\mathrm{NAD}(+) /$ SIRT1 Axis. Cell Metab 24: 526-528.

98. Csiszar A, Tarantini S, Yabluchanskiy A, Balasubramanian P, Kiss T, et al (2019) Role of endothelial NAD deficiency in age-related vascular dysfunction. 316: H1253-H1266.

99. de Picciotto NE, Gano LB, Johnson LC, Martens CR, Sindler AL, et al. (2016) Nicotinamide mononucleotide supplementation reverses vascular dysfunction and oxidative stress with aging in mice. Aging Cell 15: 522-530

100. Koopman WJ, Willems PH, Smeitink JA (2012) Monogenic mitochondrial disorders. N Engl J Med 366: 1132-1141.

101. Long AN, Owens K, Schlappal AE, Kristian T, Fishman PS, et al. (2015) Effect of nicotinamide mononucleotide on brain mitochondrial respiratory deficits in an Alzheimer's disease-relevant murine model BMC Neurol 15:19.

102. Wang X, Hu X, Yang Y, Takata T, Sakurai T, et al. (2016) Nicotinamide mononucleotide protects against beta-amyloid oligomer-induced cognitive impairment and neuronal death. Brain Res 1643: 1-9.

103. Mitchell SJ, Bernier M, Aon MA, Cortassa S, Kim EY, et al. (2018) Nicotinamide Improves Aspects of Healthspan, but Not Lifespan, in Mice. 27: 667-676.e664.

104. Moon J, Kim HR, Shin MG (2018) Rejuvenating Aged Hematopoietic Stem Cells Through Improvement of Mitochondrial Function. Ann Lab Med 38: 395-401

105. Jie Song, Jing Li, Fangji Yang, Gang Ning, Limin Zhen, et al. (2019) Nicotinamide mononucleotide promotes osteogenesis and reduces adipogenesis by regulating mesenchymal stromal cells via the SIRT1 pathway in aged bone marrow. Cell Death Dis 10: 336.

106. TV A, Cell KBJ (2018) HS to Mitigate Vascular Aging: A SIRT1 Connection. 173: 8-10.

107. Gerdts J, Brace EJ, Sasaki Y, DiAntonio A, Milbrandt J, et al. (2015) SARM1 activation triggers axon degeneration locally via $\mathrm{NAD}^{+}$ destruction. 348: 453-457.
108. Zhang S, Xue X, Zhang L, Zhang L, Liu Z, et al. (2015) Comparative Analysis of Pharmacophore Features and Quantitative StructureActivity Relationships for CD38 Covalent and Non-covalent Inhibitors. Chem Biol Drug Des 86: 1411-1424.

109. Kellenberger E, Kuhn I, Schuber F, Muller-Steffner H (2011) Flavonoids as inhibitors of human CD38. Bioorg Med Chem Lett 21: 3939-3942.

110. KA Wall, M Klis, J Kornet, D Coyle, JC Amé, et al. (1998) Inhibition of the intrinsic NAD+ glycohydrolase activity of CD38 by carbocyclic NAD analogues. Biochem J 335(Pt 3): 631-636.

111. Mukhopadhyay P, Rajesh M, Cao Z, Horváth B, Park O, et al. (2014) Poly (ADP-ribose) polymerase-1 is a key mediator of liver inflammation and fibrosis. Hepatology 59: 1998-2009.

112. Fang EF, Scheibye-Knudsen M, Brace LE, Kassahun H, SenGupta T, et al. (2014) Defective mitophagy in XPA via PARP-1 hyperactivation and NAD(+)/SIRT1 reduction. Cell 157: 882-896.

113. Day K, Waite LL, Thalacker-Mercer A, West A, Bamman MM, et al. (2013) Differential DNA methylation with age displays both common and dynamic features across human tissues that are influenced by $\mathrm{CpG}$ landscape. Genome Biol 14: R102.

114. Jones PA (2012) Functions of DNA methylation: islands, start sites, gene bodies and beyond. Nat Rev Genet 13: 484-492.

115. Maegawa S, Hinkal G, Kim HS, Shen L, Zhang L, et al. (2010) Widespread and tissue specific age-related DNA methylation changes in mice. Genome Res 20: 332-340.

116. Smith ZD, Meissner A (2013) DNA methylation: roles in mammalian development. Nat Rev Genet 14: 204-220.

117. Schultz MD, He Y, Whitaker JW, Hariharan M, Mukamel EA, et al. (2015) Human body epigenome maps reveal noncanonical DNA methylation variation. Nature 523: 212-216

118. Schultz MD, He Y, Whitaker JW, Hariharan M, Mukamel EA, et al. (2016) Corrigendum: Human body epigenome maps reveal noncanonical DNA methylation variation. Nature 530: 242.

119. Levine ME, Lu AT, Bennett DA, Horvath S (2015) Epigenetic age of the pre-frontal cortex is associated with neuritic plaques, amyloid load, and Alzheimer's disease related cognitive functioning. Aging (Albany NY) 7: 1198-1211.

120. Hannum G, Guinney J, Zhao L, Zhang L, Hughes G, et al. (2013) Genomewide methylation profiles reveal quantitative views of human aging rates. Mol Cell 49: 359-367.

121. Bock landt S, Lin W, Sehl ME, Sehl ME, Sánchez FJ, Sinsheimer JS et al. (2011) Epigenetic predictor of age. PLoS One 6(6): e14821.

122. Koch CM, Wagner W (2011) Epigenetic-aging-signature to determine age in different tissues. Aging (Albany NY) 3(10): 1018-1027.

123. Weidner CI, Lin Q Koch CM, Eisele L, Beier F, et al. (2014) Aging of blood can be tracked by DNA methylation changes at just three CpG sites. Genome Biol 15(2): R24.

124. Petkovich DA, Podolskiy DI, Lobanov AV, Lee SG, Miller RA, et al (2017) Using DNA Methylation Profiling to Evaluate Biological Age and Longevity Interventions. Cell Metab 25(4): 954-960.

125. Stubbs TM, Bonder MJ, Stark AK, Krueger F, et al. (2017) Multi-tissue DNA methylation age predictor in mouse. Genome Biol 18(1): 68.

126. Han Y, Eipel M, Franzen J, Sakk V, Dethmers-Ausema B, et al. (2018) Epigenetic age-predictor for mice based on three CpG sites. Elife 24: 7.

127. Thompson MJ, Chwialkowska K, Rubbi L, Lusis AJ, Davis RC, et al. (2018) A multi-tissue full lifespan epigenetic clock for mice. Aging (Albany NY) 10(10): 2832-2854.

128. Fahy GM, Brooke RT, Watson JP, Michael S Kobor, Steve Horvath, et al. (2019) Reversal of epigenetic aging and immunosenescent trends in humans. Aging Cell 18(6): e13028. 
129. Abbott A (2019) First hint that body’s 'biological age' can be reversed Nature 573(7773): 173.

130. Trammell SA, Schmidt MS, Weidemann BJ, Redpath P, Jaksch F, et al. (2016) Nicotinamide riboside is uniquely and orally bioavailable in mice and humans. Nat Commun 10(7): 12948.

131. Martens CR, Denman BA, Mazzo MR, Armstrong ML, Reisdorph N, et al. (2018) Chronic nicotinamide riboside supplementation is welltolerated and elevates $\mathrm{NAD}^{+}$in healthy middle-aged and older adults. Nat Commun 9(1): 1286.

132. Elhassan YS, Kluckova K, Fletcher RS, Schmidt MS, Garten A, et al. (2019) Nicotinamide Riboside Augments the Aged Human Skeletal Muscle NAD Metabolome and Induces Transcriptomic and Antiinflammatory Signatures. Cell Rep 28(7): 1717-1728.
133. Airhart SE, Shireman LM, Risler LJ, Anderson GD, Nagana Gowda GA, et al. (2017) An open-label, non-randomized study of the pharmacokinetics of the nutritional supplement nicotinamide riboside (NR) and its effects on blood NAD+ levels in healthy volunteers. PLoS One 12(12): e0186459.

134. Dolopikou CF, Kourtzidis IA, Margaritelis NV, Vrabas IS, Koidou I et al. (2019) Acute nicotinamide riboside supplementation improves redox homeostasis and exercise performance in old individuals: a doubleblind cross-over study. Eur J Nutr 6.

135. Tsubota K (2016) The first human clinical study for NMN has started in Japan. NPJ Aging Mech Dis 2: 16021.

136. Nacarelli T, Lau L, Fukumoto T, Zundell J, Fatkhutdinov N, et al. (2019) $\mathrm{NAD}^{+}$metabolism governs the proinflammatory senescenceassociated secretome. Nat Cell Biol 21(3): 397-407 